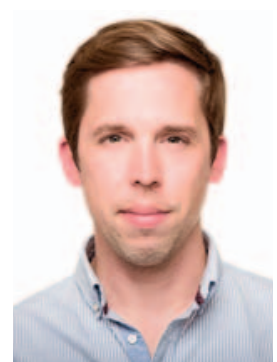

Matthias Raspe

Koordination «PneumoCampus»

\section{«Wer bin ich?» Kluge Diagnostik, achtsame Kommunikation, klare Organisation}

Herzlich willkommen zur vierten und letzten Ausgabe des «PneumoCampus» in diesem Jahr! In der letzten Ausgabe hatten wir Sie in unsere neue Reihe «Blickdiagnosen» eingeführt. Auch in dieser Ausgabe haben wir einen spannenden Fall für Sie aufbereitet, der sich am Themenschwerpunkt des Heftes orientiert. Welche Diagnose vermuten Sie? Die Auflösung finden Sie auf der Webseite von Karger Kompass Pneumologie.

Eine Diagnose ist nur die halbe Miete. Die weitere Herausforderung ist: Wie sage ich es meinem Patienten? Die Qualität der Kommunikation ist bereits vor der Diagnose ein wichtiger Baustein eines vertrauensvollen Arzt-Patienten-Verhältnisses und damit für den Behandlungserfolg essentiell. Eine professionelle Kommunikation kann erlernt und geübt werden. Schon im Studium sollten entsprechende Lerneinheiten fest im Stundenplan verankert sein. An der Universität Würzburg besteht bereits seit 10 Jahren ein Schauspielpatienten-Programm, das die beiden Koordinatoren Dr. Matthias Lukasczik und Dipl.-Psych. Hans-Dieter Wolf in dieser Ausgabe vorstellen.

Zu den Tücken der Kunst der Verständigung stand uns die Kommunikationstrainerin Dr. Gerlinde Kempendorff-Hoene in drei Fragen Rede und Antwort. Darin werden geschlechterspezifische Unterschiede in der Kommunikation im Krankenhaus deutlich, die auf ein tiefer liegendes Problem hinweisen.

Im abschließenden Beitrag stellt Ihnen Clara Vollmer vom Universitätsklinikum SchleswigHolstein in einer kurzen Rezension das Buch «Time and Life Management for Medical Students and Residents» von Michael Sabel, erschienen im Thieme Verlag, vor. Der Autor folgt - geschlechtsneutral - in seinem Werk der zentrale Frage «Wie lerne ich, mich selbst zu organisieren und meine Ziele zu erreichen?»

Wir wünschen Ihnen eine anregende Lektüre! Mit besten Grüßen,

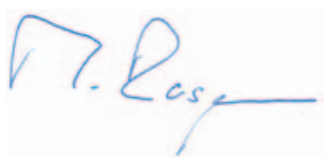

\section{KARGER}

Fax +497614520714 information@karger.com www.karger.com (c) 2017 S. Karger GmbH, Freiburg
Dr. med. Matthias Raspe

Medizinische Klinik

Schwerpunkt Infektiologie und Pneumologie

Charité - Universitätsmedizin Berlin

Augustenburger Platz 1, 13353 Berlin, Deutschland matthias.raspe@charite.de 


\section{Blickdiagnosen}

\section{Welche Diagnose vermuten Sie?}

Ein 56-jähriger Patient stellt sich aufgrund langsam zunehmender Belastungsdyspnoe beim Hausarzt vor. Der sehr sportliche Patient absolvierte bisher 1-2 Marathon-Läufe im Jahr und hatte über die letzten Monate eine langsame Abnahme seiner körperlichen Leistungsfähigkeit bemerkt. Zuletzt waren zusätzlich zur Dyspnoe ein trockener Husten, ein ungewollter Gewichtsverlust von $3 \mathrm{~kg}$ über etwa 8 Wochen und intermittierend subfebrile Temperaturen hinzugekommen. Vorerkrankungen bestehen nicht, ebenso ist keine regelmäßige Medikamenteneinnahme zu verzeichnen. Der Patient ist Nieraucher. Eine Behandlung mit einem Antibiotikum (Doxycyclin) war über den Hausarzt ohne Besserung der Beschwerden versucht worden. Daraufhin wurde ein Röntgenthorax durchgeführt, mit dem der Patient in unserer Ambulanz zur weiteren Abklärung vorgestellt wurde. Bei Vorstellung waren die Vitalparameter inklusive Atemfrequenz und peri- pherer Sauerstoffsättigung unter Ruhebedingungen im Normbereich. Auskultatorisch und perkutorisch waren im rechts mittigen bis basalen Hemithorax ein fehlendes Atemgeräusch mit gedämpftem Klopfschall zu erheben. Laborchemisch fielen moderat erhöhte Entzündungsparameter auf (Leukozyten 14,5/nl, Ref. $<10,5$ und CRP 75mg/l, Ref. <5). In der Spirometrie zeigte sich der führende Befund einer Restriktion mit reduzierter Vitalkapazität von 3,05I/65\%.

\section{Auflösung online unter: www.karger.com/blickdiagnose-kkp-4-2017}

Kontaktadresse: Herrn Dr. med. Matthias Raspe, Medizinische Klinik, Schwerpunkt Infektiologie und Pneumologie, Charité - Universitätsmedizin Berlin, Augustenburger Platz 1, 13353 Berlin, Deutschland, matthias.raspe@charite.de

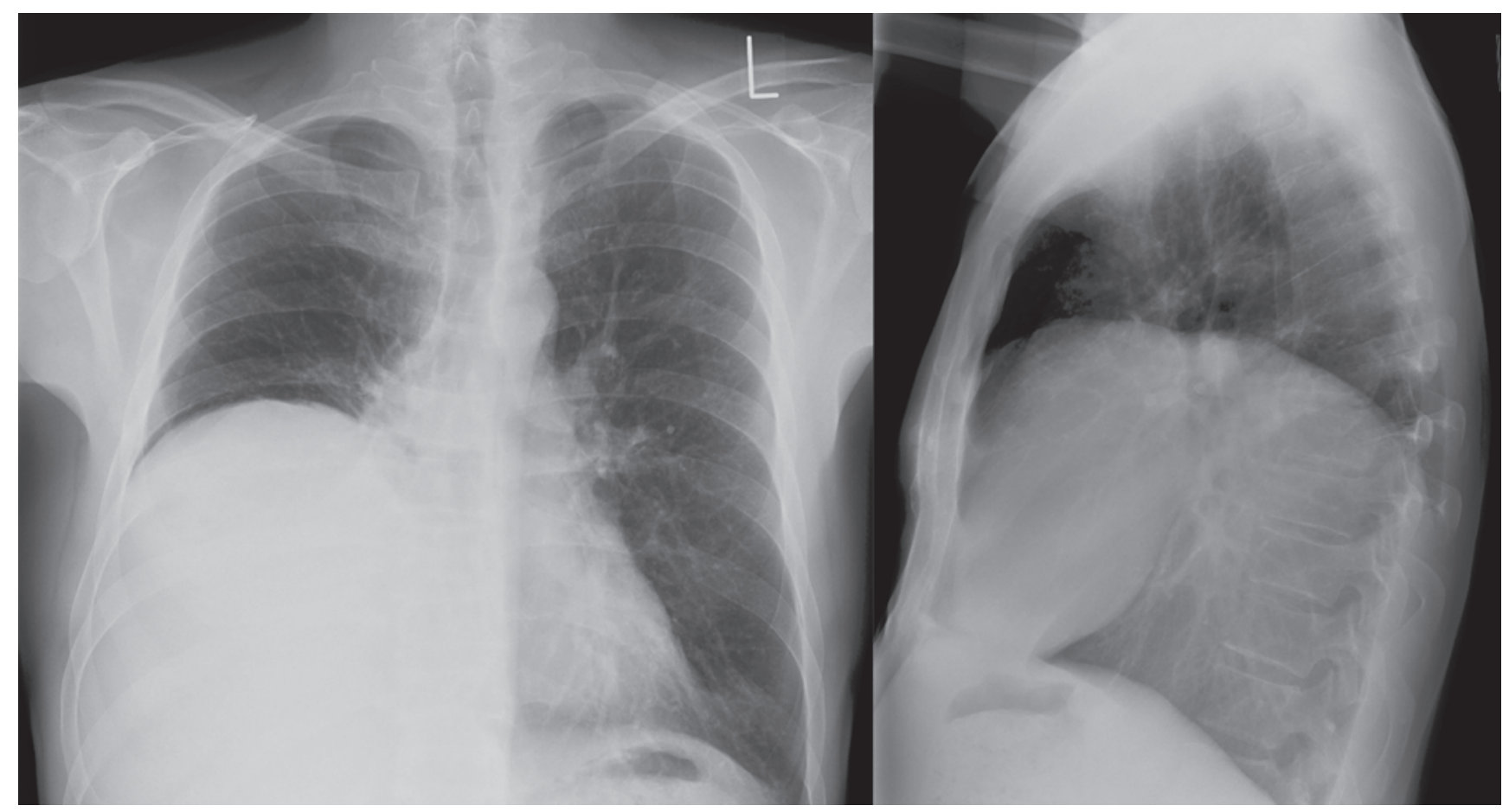

Bildbefunde mit freundlicher Genehmigung von Dr. med. C. Althoff, Leitung Interventionsradiologie Charité Campus Mitte, Institut und Klinik für Radiologie CCM und CVK, Charité - Universitätsmedizin Berlin.

\section{Arzt-Patienten-Kommunikation Schauspielpatienten-Programm an der Medizinischen Fakultät der Universität Würzburg}

Krankheitsverlauf, Adhärenz, Behandlungserfolg und nicht zuletzt eine gelungene Kommunikation [1] sind Aspekte, die eine gute Arzt-PatientenBeziehung positiv beeinflussen. Um den Grundstein für diese essenziellen kommunikativen Fähigkeiten möglichst bereits im Medizinstudium zu legen, werden weltweit an zahlreichen Universitäten Schauspielpatienten eingesetzt, d.h. Laienschauspieler, die für bestimmte Patientenrollen trainiert werden: Dies umfasst nicht nur eine medizinische Vorgeschichte mit Symptomen, Voruntersuchungen usw., sondern auch nicht-medizinische Informationen wie den Beruf oder die familiäre Situation des erdachten Patienten. Ziel ist es, einen möglichst authentischen Patienten entstehen zu lassen. Seit 2007 gibt es das Schauspielpatienten-Programm an der Universität Würzburg. Wir hatten die Möglichkeit, mit Dr. Matthias Lukasczik und Dipl.-Psych. Hans-Dieter Wolf, den beiden Koordinatoren des Programms, zu sprechen. 


\section{Wie läuft ein Schauspielpatientengespräch ab?}

Die Gespräche finden in Kleingruppen von maximal 12 Personen statt und dauern jeweils ca. 10 min. Sie werden in den Lehrveranstaltungen vorbereitet, d.h. die Dozenten erarbeiten mit den Studenten allgemeine Informationen und geben Input zur Situation und zu den Fertigkeiten, die für ein gutes Gespräch wichtig sind. Außerdem erhalten die Studenten eine schriftliche Kurzinformation mit Angaben zum Patienten, zu medizinischen Fakten und zu ihrer Aufgabe als Arzt im Gespräch.

Im Gespräch selbst sind jeweils ein Dozent bzw. Tutor sowie die anderen Mitglieder der Kleingruppe anwesend. Nach dem Gespräch folgt ein strukturiertes Feedback: Nachdem der Student, der die Arztrolle übernommen hat, seine Einschätzung des Gesprächs abgegeben hat, äußert sich der Schauspielpatient (SP) dazu, was im Gespräch gut gelaufen ist und an welchen Stellen es Verbesserungsbedarf gibt. Danach geben der Dozent und die anderen Kursteilnehmer Rückmeldung.

\section{Wie wird eine Patientenrolle kreiert?}

Zunächst überlegen wir uns, wo Bedarffür eine neue Rolle besteht. Hieraus leiten sich in der Regel schon einige Vorgaben ab (Erkrankung, Lebensstil des Patienten, vermutliche Gefühlslage usw.).

Mithilfe dieses Grundgerüstes folgt in der Regel zuerst eine Art Brainstorming mit den für das jeweilige Fach verantwortlichen Dozenten. An der detaillierten Formulierung der Rolle sind immer mehrere Personen aus verschiedenen Berufsgruppen (Psychologen, Ärzte) beteiligt.

Die Rolle umfasst im Ergebnis dann mindestens die folgenden Informationen:

- Angaben zur Person (Name, Alter inkl. Geburtsdatum, Familie)

- Angaben zur beruflichen Situation und Laufbahn

- Angaben zur aktuellen medizinischen Situation

- Angaben zur medizinischen Vorgeschichte

- Angaben zu Persönlichkeit und sozialer Situation
Die so entstandene Rolle wird dann mit einem oder mehreren SP durchgearbeitet und im Rollentraining praktisch getestet. Input von Schauspielerseite wird aufgegriffen, mögliche Unklarheiten oder «Stolpersteine» werden berücksichtigt und die Rolle entsprechend angepasst. Erst dann kann sie in der Lehrroutine eingesetzt werden.

Gibt es «schwierige» Rollen, die beispielsweise den kulturellen Hintergrund und die damit verbundenen Herausforderungen berücksichtigen?

Es gibt Rollen, die aufgrund ihrer medizinischen Situation herausfordernd sind - dazu zählt z.B. die Rolle einer jungen Mutter mit fortgeschrittener Krebserkrankung, die aller Wahrscheinlichkeit nach ihr Kind nicht aufwachsen sehen wird. Andere sicher sinnvolle und spannende Konstellationen, z.B. Angehörigengespräche oder Rollen aus anderen kulturellen Hintergründen, sind Teil unserer langfristigen Planungen, bislang aber noch nicht umgesetzt.

Welche Problemfelder/Herausforderungen haben sich im Laufe der Jahre herauskristallisiert und was kann man daraus für die Praxis lernen?

Eine der größten Schwierigkeiten, mit der gerade junge Mediziner zurechtkommen müssen, ist der immer größer werdende Zeitdruck im ärztlichen Berufsalltag. Eine der großen Herausforderungen ist es daher, zu zeigen, dass man auch in zehn Minuten eine gute Beziehung zu einem Patienten aufbauen und auf seine Bedürfnisse eingehen kann.

Aufgrund der demographischen Entwicklung nimmt auch die Arbeit mit älteren Menschen einen immer größeren Raum in der ärztlichen Arbeit ein. Deshalb sind mehrere Rollen im Würzburger SPProgramm bereits auf ältere Patienten zugeschnitten, um den Studierenden das Üben alltagsrelevanter Gesprächssituationen zu ermöglichen.

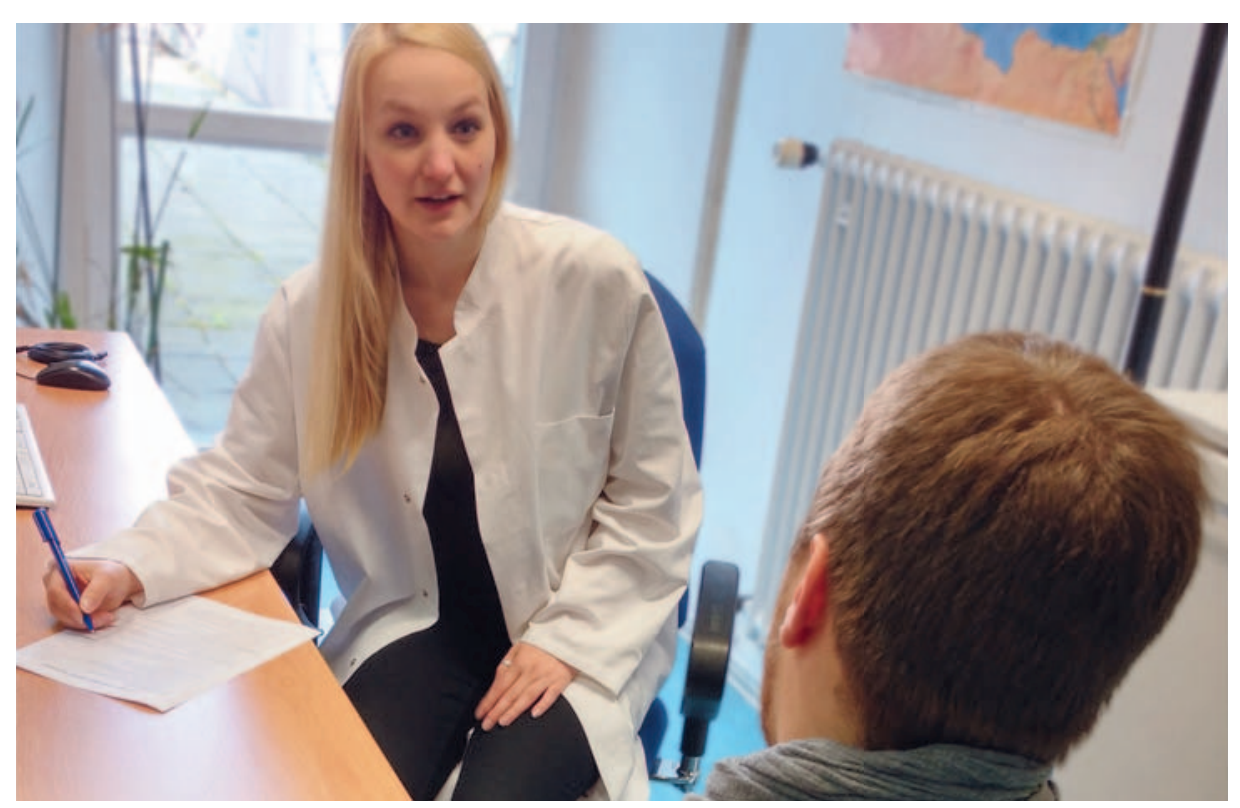

${ }^{\circ}$ Matthias Lukasczik, Schauspielpatienten-Programm Würzburg.
Welche Entwicklungen gab es in den nunmehr 10 Jahren, in denen das Programm schon läuft und welche Pläne gibt es für die Weiterentwicklung des Programms?

In erster Linie ist das Programm ausgebaut und erweitert worden - sowohl was den Pool an Rollen als auch was die Zahl der Lehrveranstaltungen mit SP-Einsätzen betrifft. Mittlerweile ist die Arbeit mit SP ein fester Bestandteil der medizinischen Lehre in Würzburg und wird von den Studenten sehr gut bewertet. Der hohe Zuspruch zeigt sich auch an den vielen Interessierten, die sich als Tutoren bzw. als SP bewerben. Die gute Entwicklung des Programms findet fächerübergreifend Anerkennung und wurde 2011 mit dem AlbertKölliker-Lehrpreis der Medizinischen Fa- 
kultät ausgezeichnet. Ein Faktor, der für die medizinische Ausbildung sicher relevant ist, ist der Nationale Kompetenzbasierte Lernzielkatalog Medizin (NKLM). Dort wird Kommunikationsfertigkeiten ein großer Stellenwert beigemessen. Daher wird sicher die Frage nach der Prüfbarkeit der erlernten Kommunikationskompetenzen, z.B. im Rahmen von OSCE-Prüfungen, noch mehr in den Vordergrund rücken. Hier steht das SP-Programm in Würzburg noch in der Planungsphase.

\section{Wir danken Ihnen herzlich für das Interivew!}

\section{Literatur}

1 Interview mit Samy Molcho: Von Mensch zu Mensch statt von Arzt zu Patient. Karger Kompass Dermatol 2017:5;98.

Kontaktadresse: Dr. Matthias Lukasczik / Dipl.-Psych. Hans-Dieter Wolf, JuliusMaximilians-Universität Würzburg, Abteilung für Medizinische Psychologie, Klinikstraße 3, 97070 Würzburg, Deutschland, matthias.lukasczik @ uniwuerzburg.de; hans.wolf@uni-wuerzburg.de.

\section{Angaben zum Schauspielpatienten-Programm in Würzburg}

Das Programm wurde 2007 von Ärzten und Psychologen des Uniklinikums (Palliativmedizin) und der Universität (Medizinische Psychologie) initiiert, damals noch beschränkt auf den Bereich Onkologie. Aktuell werden Schauspielpatienten in vier Lehrveranstaltungen eingesetzt: «Medizinische Psychologie» (Anamnese, Motivierung zur Lebensstiländerung, Aufklärung/ Diagnosemitteilung), «Praktische Klinische Untersuchungsmethoden» (Anamnese), «Kommunikationstraining Interdisziplinäre Onkologie» (Diagnosemitteilung) und «OP-Aufklärung». Insgesamt kommen derzeit ca. 30 Schauspielerinnen und Schauspieler in 20 verschiedenen Rollen zum Einsatz.

Nähere Informationen finden Sie unter www.psychotherapie.uni-wuerzburg.de/schauspielpatienten

\section{Arzt-Arzt-Kommunikation}

«Frauen reden anders, Männer auch. Gut zu wissen.»

Dr. Gerlinde Kempendorff-Hoene liegt das Thema Kommunikation sehr am Herzen. Aus diesem Engagement heraus gründete sie 2012 das «Kempendorff Privatinstitut für Kommunikation und Kultur» (KIKK). Als Coach für Sprechkultur und Auftrittskompetenz ist sie seit 2012 unterwegs und das gibt ihr regelmäßig die Möglichkeit, in verschiedene Fachgebiete hineinzuschnuppern - unter anderem auch in die Medizin.

\section{Sie empfehlen für den zwischenärztlichen Kontakt das 5-Säu-} len-System der Rhetorik. Was ist das Besondere diesem System?

Das 5-Säulen-System der Rhetorik von Christian Rangenau ordnet einfach mal alles, was - übrigens fachübergreifend - zur gelingenden Kommunikation gehört, in ein übersichtliches System. Daraus ergibt sich für den Laien erst einmal das Verständnis dafür, worauf geachtet werden muss und was gearbeitet, geübt und damit auch geändert werden kann. Vielen Menschen ist nicht klar, dass die Rhetorik ein eigenes Fachgebiet ist. Damit lässt sich ins

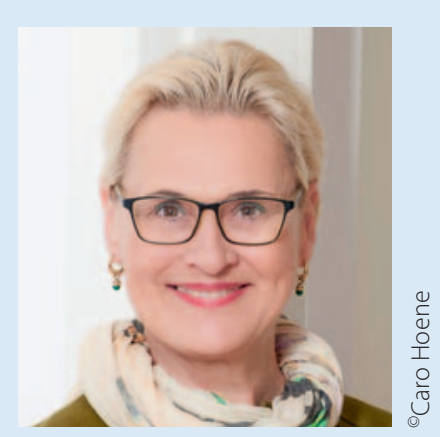

Dr. Gerlinde Kempendorff-Hoene

\section{Zur Person}

Nach ihrer freiberuflichen Tätigkeit als Kabarettistin, Sängerin, Moderatorin und Dozentin arbeitete Frau Dr. Gerlinde Kempendorff-Hoene seit 1991 als Dozentin an der Universität der Künste Berlin. 2012 machte sie sich mit der Gründung des Kempendorff Privatinstitut für Kommunikation und Kultur (KIKK) selbstständig. Seit über 30 Jahren in der Erwachsenenqualifizierung tätig, konzentriert sie sich seit 5 Jahren auf die Arbeit als Coach für Auftrittskompetenz und Sprechkultur.
Bewusstsein rücken, dass man durchaus eine «berufliche» und eine «private» Sprache pflegen kann, wobei in der ersteren gegenseitiger Respekt, Wertschätzung und Sachlichkeit immer im Vordergrund stehen müssen.

\section{Auf welche geschlechtsspezifischen Unterschiede in der Kommu- nikation sollte man gerade im medizinischen Arbeitsalltag achten?}

Die Sprache ist immer im Wandel, und mit der Gleichstellung von Mann und Frau in der Sprache geschieht gerade eine größere Veränderung, als mancher vertragen kann. Dazu kommen auch Übertreibungen, die Sachverhalte verwässern. Ich finde es angenehm, wenn man z.B. in Vorträgen oder Veröffentlichungen Gattungs- und Genusbegriff klar trennt und das im Vorfeld deutlich macht. Der Gattungsbegriff ist «Mensch», der Genusbegriff weiblich, männlich oder sächlich, d.h. wenn ich sage: «Der Mensch hat unterschiedliche Arten zu kommunizieren», muss ich nicht hinzufügen «die Menschin», denn der Begriff Mensch beinhaltet alle Geschlechter. Wenn ich aber sage: «Chefarzt Bettina Müller», sollte sich jeder daran gewöhnen, den weiblichen Begriff «Chefärztin» zu benutzen. Auch die - leider immer noch gängige - Anrede von PatientInnen mit «Was haben wir denn?» sollte langsam mal unterlassen werden. Ich antworte dann immer: «Was Sie haben, weiß ich nicht, aber ich habe ...».

Welche Kommunikationstipps geben Sie Ärztinnen, die am Beginn ihrer Karriere stehen, mit auf den Weg?

Kleine Geschichten aus dem Arbeitsalltag machen das sehr deutlich.

- Eine junge Assistenzärztin war bei mir im Training, weil sie sich über ihren Chef geärgert hatte oder viel mehr über sich und ihre Hilflosigkeit. Während der Visite begegnete der Ärzteschar auf dem Gang eine ältere Patientin, die offensichtlich Hilfe benötigte, und der Chefarzt sagte zu der Patientin, mit einem Kopfnicken zur Assistenzärztin 
deutend: «Die Anne bringt sie mal auf das Zimmer!» Damit war den anderen Kolleglnnen klar, dass er sie nicht als Kollegin wahrnimmt und der Patientin wurde vermittelt, dass sie eine Schwester sein muss. Und: Sie hat sich nicht getraut, dem Chef freundlich aber bestimmt diesen Fauxpas aufzuzeigen, den er wahrscheinlich gar nicht gemerkt hatte. Viele, gerade junge MedizinerInnen haben im immer noch sehr hierarchischen Denken (auch von PatientInnenseite) Akzeptanzschwierigkeiten. Das muss sich ändern. Dazu brauchen sie rhetorisches Geschick, das man erlernen kann.

- Eine junge Augenchirurgin erzählte mir, dass oft PatientInnen zu ihr sagen: «Wann kommt denn nun der Doktor?!». Sie war dann immer ärgerlich und wir haben erarbeitet, dass sie von nun an lächelnd sagt: «Der bin ich, und was Besseres kann ihnen nicht passieren.»
Gerade Frauen haben oft das Problem, sich emotional angegriffen zu fühlen, wo ihnen eigentlich nur nachlässiges und/oder altmodisches Denken entgegentritt. Das kann man sich abtrainieren, damit die eigene Zufriedenheit wächst und Job und Karriere gedeihen können.

\section{Frau Dr. Kempendorff-Hoene, vielen Dank für dieses Gespräch!}

\section{Weiterführende Literatur}

Kempendorff-Hoene G: Lehrer und Kabarettisten, ed 1. Berlin, Lehmanns Media, 2010.

Kontaktadresse: Dr. phil. Gerlinde Kempendorff-Hoene, Büro Kempendorff, Leibnizstraße 35, 10625 Berlin, Deutschland, www.sprechkultur-kikk.de.

\section{Buchezension}

Michael Sabel: Time and Life Management for Medical Students and Residents

Stuttgart, Thieme, 2017, 76 Seiten, 24,99 EUR

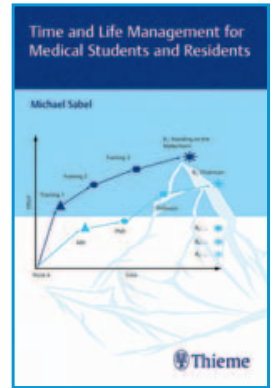

Das Buch von Herrn Sabel beschäftigt sich mit einem Thema, das im Medizinstudium leider keinen Platz gefunden hat und doch sehr wichtig ist. Die zentrale Frage lautet: Wie lerne ich mich selbst zu organisieren und meine Ziele zu erreichen?

Das Buch beginnt mit der Aufforderung, sich erst einmal über seine Ziele und Ambitionen klar zu werden. Hierzu werden verschiedene kleine Übungen und Denkexperimente vorgeschlagen. Das Buch wird erst dann zu einer wertvollen Hilfe, wenn diese Übungen auch tatsächlich durchgeführt werden, denn alle folgenden Kapitel setzen klar gesteckte Ziele voraus. Ist man sich über seine Ziele bewusst geworden, so geht es im zweiten Abschnitt darum, wie man diese erreichen kann. Wie kann ich mich motivieren meine Ziele zu verfolgen und nicht aufzugeben? Wie entscheide ich, welche Aufgaben wirklich wichtig sind? Es geht in diesem Buch um das, was wohl die meisten Medizinstudenten nach dem Studium qualvoll lernen müssen: den Alltag auf Station effektiv zu gestalten, sich auch einmal abzugrenzen und die nötige Balance zwischen Arbeits- und Privatleben zu finden. Konkrete Vorschläge, wie man seine Effektivität dann tatsäch- lich erhöhen kann, liefert das Buch meiner Meinung nach leider nur eingeschränkt, bleibt es doch häufig bei allgemeinen Grundsätzen. Die vielen Graphiken verdeutlichen die im Text beschriebenen Theorien sehr plastisch, liefern aber keine neue Aussage und waren mir zu trivial. Für Menschen, die sich bereits mit dem Thema «Selbstmanagement» beschäftigt haben, mag das Buch augenscheinlich keine neuen Erkenntnisse bieten. Außerdem fehlte mir ein stringenter Aufbau. Alle Kapitel waren zwar in sich schlüssig aufgebaut, jedoch fehlte der übergeordnete Zusammenhang zwischen den Kapiteln.

Trotz allem hat das Buch seine Daseinsberechtigung, denn eines tut es sicher: Es regt zum Denken an. Was für ein Arzt möchte ich sein? Was für ein Mensch möchte ich sein? Wo liegen meine Prioritäten? Sind meine Ziele realistisch und nutze ich meine Zeit sinnvoll? Sich bereits vor Arbeitsbeginn einmal mit diesen Fragen auseinanderzusetzen spart in der ohnehin stressigen Anfangszeit sicherlich viel Zeit und Kraft.

Clara Vollmer, Kiel

Kontaktadresse: Clara Vollmer, Klinik für Innere Medizin IV, Nieren- und Hochdruckkrankheiten, Universitätsklinikum Schleswig-Holstein, Schittenhelmstraße 12, 24105 Kiel, Deutschland, claracvollmer@googlemail.com. 